https://creativecommons.org/licenses/by/4.0/

\title{
TECNOLOGÍAS DE COMUNICACIONES DE LÍNEA DE ALIMENTACIÓN PARA REDES INTELIGENTES: UNA EVALUACIÓN DEL ESTADO DE LA TÉCNICA
}

\author{
Power line communications technologies for smart grids: \\ an evaluation of the state of the art
}

LUIS ALEJANDRO MOTTA ${ }^{1}$, JUAN DAVID GONZÁLEZ ORJUELA², EFRAIN BERNAL ALZATE ${ }^{3}$

Recibido:22 de octubre de 2018. Aceptado:08 de noviembre de 2018

DOI: http://dx.doi.org/10.21017/rimci.2019.v6.n11.a55

\begin{abstract}
Resumen
En este artículo se realiza una evaluación del estado de la técnica aplicada a Smart Grid. El análisis aborda los conceptos básicos, identificando el funcionamiento de cada tipo de tecnología de Power Line Communications (PLC) y comparando los módulos actuales disponibles en el mercado. El resultado de este análisis nos permite sugerir una tecnología para la realización de PLC dentro del concepto actual de Smart Grid.
\end{abstract}

Palabras clave: Smart Grid, Power line communications, medidor de inteligencia, estado del arte.

\begin{abstract}
In this article an evaluation of the state of the art applied to Smart Grid is carried out. The analysis addresses the basic concepts, identifying the operation of each type of Power Line Communications (PLC) technologies, and comparing the current modules available in the market. The result of this analysis allows us to suggest a technology for the realization of PLC within the actual Smart Grid concept.
\end{abstract}

Keywords: Smart Grid, Power line communications, smart meter, state of the art.

\section{INTRODUCCIÓN}

L OS SISTEMAS de distribución están compuestos por una gran variedad de elementos conectados en la red, algunos de ellos son los generadores distribuidos tanto a pequeña como a gran escala, autogeneradores, fuentes convencionales, fuentes no convencionales y gran cantidad de usuarios conectados lo cual caracteriza una red de baja tensión.

En la operación de los sistemas de distribución, debido a su complejidad topológica, están expuestos a variedad de fallas las cuales comprometen el su- ministro de energía. Estos problemas en el sistema son complejos y difíciles de detectar, debido a que la red es muy extensa y de diferentes topologías (aéreas o subterráneas) que están expuestas, por lo que hay alta probabilidad de que ocurran fallas, las cuales comprometen los índices de calidad del servicio.

Las redes de distribución cuentan con la necesidad de digitalización de la energía que permita obtener una medición más precisa y confiable. En estos sistemas la detección de fallas en el suministro es un factor complicado de obtener, por la complejidad topológica de la red que

1 Facultad de ingeniería de la Universidad de la Salle Bogotá, Colombia. Correo electrónico: lmotta12@unisalle.edu.co

2 Facultad de ingeniería Universidad de la Salle Bogotá, Colombia. Correo electrónico: jgonzalez59@unisalle.edu.co

3 Facultad de ingeniería Universidad de la Salle Bogotá, Colombia. ORCID: https://orcid.org/0000-0002-9093-8224 Correo electrónico: ebernalal@unisalle.edu.co 
afectan la confiabilidad y la seguridad, debido a las demoras en los tiempos de la detección ya que los usuarios son los que tienen que reportarla, para que el operador de red logre identificarla y así tome una decisión que muchas veces no es la más oportuna para obtener un control de esta. Debido a la gran cantidad de usuarios conectados, es más difícil tener una supervisión constante de la condición en la que se encuentra cada usuario, ya que los sistemas son susceptibles a variaciones y desviaciones en los patrones de consumo. A medida que haya un sistema que permita tener un monitoreo centralizado, admite tener una operación del sistema más ágil, confiable y amplia. Circunstancias que pueden atenderse de forma más precisa si la red dispone de un sistema de comunicación.

A causa de los problemas que se han presentado en los sistemas de distribución, se han desarrollado alternativas para la solución de dichos inconvenientes mediante la implementación de la tecnología PLC con la ventaja de que la red ya existe se pretende transmitir información por medio de la red eléctrica[1]. En los sistemas de potencia existe un interés en el desarrollo de los sistemas de comunicación entre los agentes del mercado, además existe la necesidad de medir y transmitir datos. Por lo tanto, es oportuno y apropiado profundizar en la historia temprana del campo. Entre las primeras aplicaciones de comunicación que utilizan líneas eléctricas[2].

La comunicación a través de las líneas de alta tensión (PLC) es una idea antigua que se remonta a principios de la de cada de 1900, cuando las primeras patentes fueron presentadas en esta área. Desde entonces, las empresas eléctricas de todo el mundo han utilizado esta tecnología para mediciones remotas y control de carga. El incentivo para utilizar PLC es que la red eléctrica proporciona una infraestructura que es bastante extensa y dominante que cualquier otra alternativa con cable/ inalámbrica, por lo que casi todos los dispositivos con conexión a la red eléctrica pueden ser servicios de valor agregado[3].

Las tecnologías Smart Grid (SG) están atrayendo una atención creciente debido a su capacidad inherente para realizar un sistema de gestión de energía sostenible mediante el uso de redes inteligentes para futuros prospectos. Las infraestructuras de SG requieren una técnica/plataforma de comunicación bidireccional e interactiva entre la red eléctrica y los consumidores para mejorar la eficiencia energética, seguridad y fiabilidad de los sistemas de generación, transmisión y distribución[4]. Para este propósito, las comunicaciones de línea eléctrica (PLC) han demostrado ser una alternativa factible, que ofrece promesas favorables para comunicaciones de extremo a extremo, desde el nivel de electrodomésticos, aplicaciones de monitorización/ - control hasta gestión basada en generación distribuida (DG) en aplicaciones de control con una red existente 'gratuita' [1] [5]. Se prevé que el mercado mundial de PLC superará los siete mil millones en el 2020 por sus aplicaciones en la infraestructura de medición avanzada $(\mathrm{AMI})^{4}$, sistemas remotos de telemetría, equipos de acceso a internet de banda ancha de alta velocidad, la automatización de vehículos, comunicaciones de un lado al otro, hogares inteligentes, carga de vehículos eléctricos, así como el micro inversor para fuentes alternativas de energía, iluminación y temperatura monitor/control[6].

El objetivo de este proyecto es investigar las aplicaciones, el funcionamiento y los tipos de PLC que existen actualmente para su utilización en las SG, enfocados en las redes de distribución (LV). Identificar cual de todos los PLC existentes es más viable para la realización e implementación de un modelo de Smart Grid con este tipo de tecnología.

Con base a este objetivo y lo establecido se realizarán los indicadores de evaluación para elegir la tecnología mas adecuada para la implementación de un modelo de Smart Grid, en donde se observarán antecedentes, características, casos de éxito y módulos existentes.

\section{CRiterios De eVAluación}

Se realizará una serie de comparaciones para tener más claro la tecnología a elegir de este modo se revisarán criterios como lo son:

4 La infraestructura de medición avanzada (AMI) es un sistema integrado de medidores inteligentes, redes de comunicaciones y sistemas de administración de datos que permite la comunicación bidireccional entre los servicios públicos y los clientes. 
Antecedentes de la tecnología.

- Proyectos realizados con las Power Line Comunications.

- Clasificación de la tecnología.

- Características técnicas de las tecnologías PLCc omo lo son: El rango de velocidad, tipo de comunicación, frecuencia, balance de ruido, consumo de energía, distancia y costo.

- Módulos más utilizados.

De esta manera y con la evaluación que se realizará se podrá elegir la tecnolog ía mas adecuada, para diseñar un modelo de Smart grids con PLC.

\section{Antecedentes EN LA COMUNICACIÓN POR PLC}

Algunos de los principales desarrollos relacionados con las tecnologías de redes inteligentes se sitúan en Australia, Canadá, Europa, Brasil, Estados Unidos, China, Japón y Corea del Sur[7].

\section{III-A. Inicios en la comunicación por PLC}

- En 1838, Edward Davy propuso la medición remota de las fuentes de alimentación con el propósito de verificar los niveles de tensión de las baterías en sitios donde no hubiera operadores en el sistema de telégrafo de Londres Liverpool.

- En 1897, Joseph Routin y C.E.L. Brown patentaron en Gran Bretaña su medidor de electricidad el cual enviaba las señales a través de las líneas de potencia.

- Chester Thoradson de Chicago patentó su sistema para la lectura remota de medidores de electricidad en 1905. Este sistema no fue muy utilizado dado sus pocos beneficios.

- La Carrier Frecuency Transmision (CTS) de voz sobre redes de transmisión de alta tensión comenzó en 1920. La amplia red ofreció un canal de comunicación bidireccional por ejemplo entre estaciones de transformación y plantas de energía, Debido a las características de transmisión favorables, los niveles de ruido bajos y frecuencias de la portadora relativamente altas $(15 \mathrm{kHz}-500$ $\mathrm{kHz}$ ), la distancia máxima entre transmisor y receptor era máximo $900 \mathrm{~km}$ con una potencia de 10W[8].

- En 1950 se empezó con una frecuencia de $10 \mathrm{~Hz}$ y $10 \mathrm{~kW}$ de potencia, con comunicación en un solo sentido, aplicándose para el control de líneas eléctricas y para el control remoto de relés.

- A mediados de 1980 se inició la investigación sobre el uso de la red eléctrica para soportar la transmisión de datos, en bandas de entre 5 - $500 \mathrm{kHz}$, siempre en una sola dirección. Mas adelante, las empresas eléctricas empezaron a utilizar sus propias redes para la transmisión de datos de modo interno.

- En 1997 se realizaron las primeras pruebas para la transmisión bidireccional de señales de datos a través de la red eléctrica y el inicio de la investigación de Ascom (Suiza) y Norweb (Reino Unido).

- En 2000 se llevaron a cabo las primeras pruebas en Francia por EDF R\&D y Ascom[9].

\section{III-B. Proyectos en Smart Grid}

Estados Unidos actualmente han realizado diversos proyectos con el fin de obtener mayor variedad en fuentes de generación, el cual incluye generación distribuida, al mismo tiempo que se aplique el uso de almacenamiento de la energía y flexibilidad en el uso de la red eléctrica entre el consumidor/ proveedor[7]. Los proyectos son Pacific Northwest Smart Grid Demonstration Project[10], Texas (Smart Texas) y Huston (Houston's Smart Grid)[11].

En Europa empresas que conforman la Iniciativa Europea de Redes Eléctricas (EEGI) como lo son Twenties, Umbrella, iTesla, EcoGrid EU y demás, trabajan en conjunto para mejorar la eficiencia energética, reducción de emisiones, integración de energías renovables y la implementación de contadores inteligentes[12]. 
Smart Grid, Smart City fue un proyecto financiado por el gobierno australiano por 100 millones USD, dirigido por AusGrid. El objetivo de este proyecto era eliminar barreras e incentivar la inversión en RI. Como perciben y responden los consumidores residenciales a las oportunidades que presentan las tecnologías RI. A pesar del esfuerzo de Australia para incentivar el uso de redes inteligentes, estas se encuentran aún en un estado muy temprano[13].

En Colombia empresas como CODENSA, EPM, Electricaribe, EPSA y EMCALI, han venido desde hace algunos años iniciativas en implementación de algún tipo de inteligencia en sus procesos y no en un marco de trabajo sectorial sino aisladas del resto de las empresas del sector[14].

EMCALI invirtió en la implementación de la tecnología PLC para la medición y control de consumos de energía, mediante la tecnología denominada TWACS. Actualmente, EMCALI tiene instalada la infraestructura en todo el sistema de distribución de EMCALI, con lo que tiene garantizada la cobertura para el 99 \% de los 600.000 usuarios del sistema.

El proyecto "Smart Metering" de Codensa, tiene como objetivo emplear y evaluar los medidores inteligentes, así como mostrarles a los clientes y al regulador los beneficios de la Infraestructura de
Medición Avanzada (AMI) en cuanto a la eficiencia energética, la calidad del suministro de energía eléctrica, el control de perdidas, la gestión remota de los medidores (lectura, suspensión, reconexión, balances de energía y reporte de eventos) y la capacidad de intercambio de información técnica[15].

\section{Clasificación de las PlC}

Actualmente las tecnologías PLC brindan una manera cómoda y fiable de medición y transmisión de datos, basadas con técnicas innovadoras, normatividad necesaria tanto para el sistema de potencia como para implementación de estas tecnologías, estas pueden clasificarse según su banda de frecuencia $(0.3 \mathrm{kHz}-250 \mathrm{MHz})$ y su rango de velocidades de transmisión de datos (100bps1.8Gbps), como se observa en el cuadro II. Las tecnologías Broad Band son dedicadas generalmente a las aplicaciones de acceso a internet también conocidas como banda ancha sobre líneas eléctricas, las tecnologías Narrow Band son típicamente usadas como distribuidoras de líneas.

\section{IV-A. Ultra Narrow Band (UNB)}

Las tecnologías Ultra Narrow Band son aquellas que operan a un rango de velocidad de transmisión de datos muy baja (aproximadamente

Cuadro I. Lista de acrónimos usados en el artículo.

\begin{tabular}{|llll|}
\hline \multicolumn{1}{|c|}{ Acrónimo } & \multicolumn{1}{c|}{ Significado } & Acrónimo & \multicolumn{1}{c}{ Significado } \\
AMI & Advanced Metering Infraestructure & LV & Low Voltage \\
AMR & Automatic Meter Reading & MV & Medium Voltage \\
AN & Access Network & NB & Narrow Band \\
BB & Broad Band & OPLC & Operator Power Line Communications \\
CDMA & Code Division Multiple Access & PHY & Physical Layer \\
CENELEC & Comité Européen de Normalisation Électrotechnique & PLC & Power Line Communications \\
CP & Control Panel & PS & PostScript \\
CPU & Central Processing Unit & QAM & Quadrature amplitude modulation \\
CTS & Clear to Send & QB & Quasi Band \\
DG & Distributed Generation & QPSK & Quadrature phase shift keying \\
DIN & Rail Terminal Blocks Relays & RI & Red Inteligente \\
HDR & High Data Rate & SG & Smart Grid \\
HF & High Frecuency & SM & Simatic Module \\
HMI & Human Machine Infraestructure & TDMA & Time Division Multiple Access \\
IEC & International Electrotechnical Commission & TIA & Telecommunicationes Industry Association \\
IEEE & Institute of & TWACS & Two-Way Automatic \\
IP & Electrical and Electronics Engineers & Internet Protocol & Communicacions System \\
ISO & International Organization for Standardization & UDP & User Datagram Protocol \\
LDR & Low Data Rate & VHF & Ultra Narrow Band \\
\hline
\end{tabular}

Rev. Ingeniería, Matemáticas y Ciencias de la Información Vol. 6 / Núm. 11 / enero - junio de 2019; pág. 35-44 
100bps), estas tecnologías permiten operar en dos rangos de frecuencia: Ultra baja $(0.3-3 \mathrm{KHz})$ o en super baja $(30-300 \mathrm{~Hz})$. Estas también cuentan con un rango operativo bastante amplio, el cual puede alcanzar los $150 \mathrm{~km}$ o incluso más[2]. Aunque si es cierto que la velocidad de los datos es bastante baja, los sistemas a los cuales se aplica esta tecnología resultan ser bastante eficientes debido a las formas de paralelización que resultan ser capaces de soportar escalabilidad. La UNB es una tecnología madura que ha estado en el campo durante varios años y han sido implementadas en diversas utilidades, lo cual es una gran ventaja a favor.

\section{IV-B. Narrowband (NB)}

Estas tecnologías operan dentro de un rango de frecuencia medio de hasta $500 \mathrm{kHz}$ y ofrece un rango de velocidad de transmisión que va desde 1 kbps hasta los $100 \mathrm{kbps}$. El comité Europeo de Normalización Electrotécnica (CENELEC) ha aprobado las frecuencias iguales y menores a $148.5 \mathrm{kHz}$ para ser implementadas en sistemas con aplicaciones en alta tensión, abarcando de media tensión a baja tensión[16]. La NB ha sido ser una solución atractiva paras las compañías de electricidad ya que les permite controlar la entrega de potencia, comunicaciones, aplicaciones para la detección de fallas y así tener facilidad en la toma de decisiones ante cualquier anormalidad. Esta tecnología también se basa en aplicaciones de la gestión de la energía eficiente a través de líneas eléctricas y de internet, la cual proporciona alta fiabilidad de la transmisión de datos dentro de su operación (hasta 500 kbps), otras de sus aplicaciones son basadas en crear soluciones para el ahorro de energía en las actividades principales de la distribución de energía a gran escala, como lo son la lectura del medidor y el control remoto del sistema de distribución[6].

En las últimas décadas, las empresas de servicios públicos han implementado varias soluciones $\mathrm{AMR}^{5}$ / AMI que utilizan PLC, conexiones ina- lámbricas y líneas telefónicas. En lo que respecta a PLC, las primeras implementaciones involucraron tecnologías UNB-PLC como Turtle System ${ }^{6[17] ~ y ~}$ TWACS [18], [19]. Ambos sistemas usan perturbaciones de la forma de onda de voltaje para la comunicación de salida (subestación a metro) y de la forma de onda actual para la comunicación de entrada (metro a subestación)[20].

\section{IV-C. Broadband (BB)}

Tecnologías que operan en las bandas de HF / VHF (1.8-250 MHz) y que tienen una tasa PHY que varía de varios megabits por segundo a varios cientos de megabits por segundo. BB- PLC se puede dividir de la siguiente manera:[21]

- Si HomePlug Green Phy se usa en cables radiantes (todos los cables, excepto los coaxiales), el rango de frecuencia está limitado a $2 \mathrm{MHz}$ hasta $28 \mathrm{MHz}$. El esquema de modulación se fija a QPSK, también conocido como 4- QAM. La velocidad de datos, que se puede lograr con este método, es de 9,8 Mbps en la línea, lo que da como resultado una velocidad de datos del usuario de 5,6 Mbps UDP.

- Si HomePlug AV se utiliza en cables radiantes (todos los cables, excepto los coaxiales), el rango de frecuencia se limita a $2 \mathrm{MHz}$ hasta $28 \mathrm{MHz}$. El esquema de modulación se adapta dinámicamente a las condiciones de la línea y varía desde QPSK hasta 1.024 QAM. Con eso, la velocidad de datos en la línea sube a $200 \mathrm{Mbps}$, lo que da como resultado una velocidad de datos del usuario de aproximadamente $95 \mathrm{Mbps}$ UDP.

- Si HomePlug AV2 / IEEE 1901 se aplica en dos cables radiantes, se usa el rango de frecuencia desde $2 \mathrm{MHz}$ hasta $68.5 \mathrm{MHz}$. El esquema de modulación se adapta diná-

5 AMR es una tecnología más antigua que solo recolecta el consumo de energía eléctrica y transfiere esa información desde el medidor eléctrico en el hogar hasta la utilidad (comunicación de una vía).

6 Turtle Systems, se basa en la comunicación de baja frecuencia, por ejemplo 5-10 Hz, a través de la línea de energía y tiene un alcance de 100 $\mathrm{km}$ y utilizan un ancho de banda reducido. Debido a que funciona a baja frecuencia las señales pueden pasar a través de diferentes niveles de transformación y de esta forma disminuyen el número de emisores.

7 TWACS es un sistema de comunicación de utilidad de red fija que utiliza tecnología patentada para comunicarse a través de líneas de energía eléctrica, que proporciona bajo costo, comunicación confiable bidireccional entre el centro de control y los consumidores de electricidad, agua y gas. 
micamente a las condiciones de la línea y varía desde QPSK hasta 4.096 QAM. Con eso, la velocidad de datos en la línea sube a 500 Mbps, lo que da como resultado una velocidad de datos del usuario de aproximadamente $250 \mathrm{Mbps}$ UDP.

- HomePlug AV2 / IEEE 1901 aplicado en tres cables utiliza la misma banda de frecuencia como en dos cables, pero desviado de los dos primeros transmisores y 2 receptores integrados en el chip y en uso. El esquema de modulación se adapta dinámicamente a las condiciones de la línea y varía desde QPSK hasta 4.096 QAM. Con la doble transmisión / recepción en tres hilos, la velocidad de datos sube a $1.200 \mathrm{Mbps}$, lo que da como resultado una velocidad de datos del usuario de aproximadamente $500 \mathrm{Mbps}$ UDP.

\section{IV-D. Quasiband (QB)}

China en los últimos años ha desarrollado la comunicación de banda ancha de línea de potencia cuasiestacionario (QB-PLC), que es una integración de NB-PLC y de banda ancha de la línea eléctrica de comunicación (BB-PLC) tecnologías, aporta ventajas en banda de frecuencia (1 witin $10 \mathrm{MHz}$ ) y suministra datos de alta velocidad de hasta 2 Mbps, para largas distancias AMI redes / sistemas[6].

\section{ANÁlisis y COMPARACIÓN}

Se tiene cuatro tipos de tecnologías que son Ultra Narrow Band (UNB), Narrow Band (NB), Quasi Band (QB) y Broad Band (BB), debido a que cada una trabaja a diferentes rangos de frecuencia y velocidades se realizará una comparación para poder determinar cuál tecnología es la más eficiente.

- Se tiene que las tecnologías de banda estrecha Ultra Narrow Band (UNB) y Narrow Band (NB), parte de sus ventajas es que su consumo de energía es mínimo, poseen un excelente balance de ruido y una alta fiabilidad en la trasmisión de datos, entre sus desventajas se encuentran que son de comunicación lenta y su costo es muy elevado esto también hecho que se busque otras alternativas para este tipo de tecnología.

- Las tecnologías de banda ancha Quasi Band (QB) y Broad Band (BB), poseen ventajas como lo son su tipo de comunicación que es muy rápido en comparación a las de banda estrecha y sus costos son medios, entre sus desventajas se encuentra que no poseen un rechazo contra el ruido y la fiabilidad depende de la calidad de la línea. Parte de las ventajas de las cuatro tecnologías es que pueden diseñarse para distancias muy largas y permiten una comunicación de datos bidireccional que ha hecho que se empiece a evaluar que tecnología es óptima para una Smart Grid.

- Narrow Band (NB), tiene una ventaja bastante amplia con respecto a los otras tres tecnologías y es que tiene la capacidad de actualizarse a cualquiera de las tecnologías esto la hace mucho más flexible al momento de ser utilizada para generar comunicaciones en Smart Grid.

- En el cuadro II se puede observar los rangos de frecuencia y diferentes velocidad de los cuatro tipos de tecnología con los cuales se puedo ver mas claro las ventajas y desventajas de cada una estas.

Según lo dicho anteriormente, la tecnología que más ventajas nos ofrece según su costo y beneficio es la NarrowBand, esta tecnología ofrece distintas aplicaciones según el sector a trabajar[22].

En las redes de alta tensión $(110 \mathrm{kV}-380 \mathrm{kV})$ permite la detección del rompimiento de un aislador, corto circuito, ruptura en el conductor, apertura y cierre de un breaker, esto se da a un monitoreo constante en tiempo real, lo que permite ser un complemento para los sistemas SCADA ${ }^{8}$ y tener una estación remota de vigilancia.

8 Un sistema SCADA (Supervisory Control And Data Acquisition) es el conjunto de software y hardware que sirven para poder comunicar, controlar y supervisar diversos dispositivos de campo así como controlar de forma remota todo el proceso [23]. 
En las redes de media tensión $(10 \mathrm{kV}-30 \mathrm{kV})$ permite la detección de fallas, monitoreo, prevenciones de aislamiento, conexión de subestaciones para el estado de los equipos y el estado del flujo de potencia, esta también permite adaptarse a la red básica para la implementación de AMI y resulta ser un soporte para la siguiente generación de distribución.

Para las redes de baja tensión (110 V-400 V) sus aplicaciones son un poco más limitadas ya que se basan principalmente en la medición inteligente, la comunicación y conexión de vehículos eléctricos en la red, y la administración del sistema de energía en un hogar o edificio.

\section{V-A. Módulos PLC}

En la actualidad las tecnologías mas utilizadas son la NB y la BB, por lo que los módulos están enfocados en ellas. En el mercado se consiguen distintos módulos según su ancho de banda y el tipo de modulación que este utiliza, en el cuadro III se clasifican los módulos más usados en la actualidad para distintas aplicaciones de comunicación de datos a través de las líneas eléctricas ya sean de transmisión, distribución o residenciales.

Con lo dicho anteriormente, la tecnología que mas ventajas ofrece es la Narrow Band y el analisis en el cuadro II, por lo que es oportuno la comparación

Cuadro II. COMPARACIÓN ENTRE TECNOLOGíAS DE PLC.

\begin{tabular}{|c|c|c|c|}
\hline $\begin{array}{c}\text { UltraNarrow } \\
\text { Band } \\
\text { (UNB) }\end{array}$ & $\begin{array}{c}\text { NarrowBand } \\
\text { (NB) }\end{array}$ & $\begin{array}{l}\text { QuasiBand } \\
\text { (QB) }\end{array}$ & $\begin{array}{l}\text { BroadBand } \\
\text { (BB) }\end{array}$ \\
\hline $\begin{array}{l}\text { Rango de } \\
\text { velocidad } \\
\text { muy bajo } \\
\text { (100-120 bps) }\end{array}$ & $\begin{array}{c}\text { Rango de } \\
\text { velocidad } \\
\text { bajo } \\
(1-100 \mathrm{kbps})\end{array}$ & $\begin{array}{c}\text { Rango de } \\
\text { velocidad } \\
\text { alto } \\
\text { ( Hasta } 2 \mathrm{Mbps} \text { ) }\end{array}$ & $\begin{array}{l}\text { Rango de } \\
\text { velocidad } \\
\text { muy al to } \\
\text { (3.8 Mbps- } \\
1.8 \mathrm{Gbps})\end{array}$ \\
\hline Banda estrecha & Banda estrecha & Banda ancha & Banda ancha \\
\hline $\begin{array}{c}\text { Comunicación } \\
\text { muy lenta }\end{array}$ & $\begin{array}{c}\text { Comunicación } \\
\text { lenta }\end{array}$ & $\begin{array}{c}\text { Comunicación } \\
\text { rapida }\end{array}$ & $\begin{array}{c}\text { Comunicación } \\
\text { muy rapida }\end{array}$ \\
\hline $\begin{array}{c}\text { Frecuencias } \\
\text { ultra bajas } \\
(30-300 \mathrm{~Hz}) \text { y } \\
\text { super bajas } \\
(0.3-3 \mathrm{kHz})\end{array}$ & $\begin{array}{c}\text { Frecuencias } \\
\text { medias } \\
(\text { Hasta } 500 \mathrm{kHz})\end{array}$ & $\begin{array}{c}\text { Frecuencias } \\
\text { altas } \\
(1-10 \mathrm{MHz})\end{array}$ & $\begin{array}{c}\text { Frecuencias } \\
\text { muy altas } \\
(1.8 \mathrm{MHz}- \\
86 \mathrm{MHz})\end{array}$ \\
\hline $\begin{array}{c}\text { Excelente } \\
\text { balance de ruido }\end{array}$ & $\begin{array}{c}\text { Cancela } \\
\text { ruido } \\
\text { de banda ancha } \\
\text { no deseado }\end{array}$ & $\begin{array}{l}\text { No tiene } \\
\text { rechazo } \\
\text { de ruido }\end{array}$ & $\begin{array}{l}\text { No tiene } \\
\text { rechazo } \\
\text { de ruido }\end{array}$ \\
\hline $\begin{array}{l}\text { Consume } \\
\text { poca } \\
\text { energía }\end{array}$ & $\begin{array}{l}\text { Consume } \\
\text { poca } \\
\text { energía }\end{array}$ & $\begin{array}{l}\text { Consume } \\
\text { poca } \\
\text { mucha } \\
\text { energía }\end{array}$ & $\begin{array}{l}\text { Consume } \\
\text { poca } \\
\text { mucha } \\
\text { energía }\end{array}$ \\
\hline $\begin{array}{c}\text { Costo muy } \\
\text { elevado }\end{array}$ & $\begin{array}{c}\text { Costo } \\
\text { elevado }\end{array}$ & $\begin{array}{l}\text { Costo } \\
\text { medio }\end{array}$ & $\begin{array}{l}\text { Costo } \\
\text { medio }\end{array}$ \\
\hline $\begin{array}{c}\text { Largas } \\
\text { distancias }\end{array}$ & $\begin{array}{c}\text { Largas } \\
\text { distancias }\end{array}$ & $\begin{array}{c}\text { Largas } \\
\text { distancias }\end{array}$ & $\begin{array}{c}\text { Largas } \\
\text { distancias }\end{array}$ \\
\hline $\begin{array}{c}\text { Comunicación } \\
\text { de datos } \\
\text { bidireccional }\end{array}$ & $\begin{array}{c}\text { Comunicación } \\
\text { de datos } \\
\text { bidireccional }\end{array}$ & $\begin{array}{c}\text { Comunicación } \\
\text { de datos } \\
\text { bidireccional }\end{array}$ & $\begin{array}{c}\text { Comunicación } \\
\text { de datos } \\
\text { bidireccional }\end{array}$ \\
\hline $\begin{array}{c}\text { Muy alta } \\
\text { fiabilidad en la } \\
\text { transmisión } \\
\text { de datos }\end{array}$ & $\begin{array}{l}\text { Alta fiabilidad } \\
\text { en la } \\
\text { transmisión } \\
\text { de datos }\end{array}$ & $\begin{array}{c}\text { La fiabilidad } \\
\text { depende de la } \\
\text { calidad } \\
\text { de la línea }\end{array}$ & $\begin{array}{c}\text { La fiabilidad } \\
\text { depende de la } \\
\text { calidad } \\
\text { de la línea }\end{array}$ \\
\hline
\end{tabular}


Cuadro III. MÓDULOS PLC.

\begin{tabular}{|l|l|}
\hline \multicolumn{1}{|c|}{ NarrowBand } & \multicolumn{1}{c|}{ BroadBand } \\
\hline X-10 [24] [25] & HomePlug Green PYH [26] \\
\hline KONNEX [27] & HomePlug AV PYH [28] \\
\hline ISO/IEC 14908-3 [29] & HD PLC [30] \\
\hline G3 PLC [31] & IEEE P1901 [32] \\
\hline PRIME [33] & G.hn [34] \\
\hline IEEE P1902 [35] & HomePlug AV2 [36] \\
\hline ITU G.hemn [37] & \\
\hline
\end{tabular}

de los mejores módulos de esta. En el cuadro IV se realiza una comparación de los módulos PRIME, G3 y PL360 lo cuales son los mas utilizados actualmente, que se calificaran con base de alto (color verde), medio (color naranja) y bajo (color rojo), donde se destacan las características mas relevantes como lo son la velocidad, la frecuencia, solidez, confiabilidad y demás aspectos importantes.

Como se observa en el cuadro IV los tres módulos se encuentran bastante bien calificados, pero hay uno que resalta de los demás. En comparación del módulo PRIME y G3, este último es el mejor calificado debido a sus grandes beneficios en cuanto a solidez de ruido, confiabilidad, corrección de error, adaptabilidad y rendimiento, lo único desfavorable en comparación a los otros dos es su precio en el mercado[38] [39]. Como también se observa en este mismo cuadro el módulo PL360 tiene sus características en su gran mayoría de color verde, este módulo tiene una particularidad especial, este permite la combinación de los dos tipos de tecnologías anteriormente mencionadas, lo que permite obtener las mejores características de cada una de las tecnologías e incorporarlas en otra[40].

\section{Conclusiones}

La tecnología PLC no es un desarrollo nuevo, es una alternativa que se ha estudiado e implementado desde hace algunos años. Esta tecnología resulta muy atractiva y una alternativa para los servicios adicionales de comunicación, además de ser un complemento para las tecnologías que ya han estado durante varios años.

Para una Smart Grid se requiere tener una alta fiabilidad en la transferencia de datos por lo que la UltraNarrowBand (UNB) y la NarrowBand (NB) son las más aptas, pero si se tiene una buena calidad en la línea se puede implementar una QuasiBand o una BroadBand para obtener buena fiabilidad en la transferencia de datos a velocidades mayores y reduciendo sus costos.

Según el cuadro II la tecnología que mejor se acomoda a las aplicaciones de comunicación por líneas de distribución es la NarrowBand debido a la alta fiabilidad que tiene en la transmisión de datos y en la facilidad de actualizarse a los nuevos sistemas de comunicación.

Cuadro IV. COMPARACIÓN DE MÓDULOS.

\begin{tabular}{|l|c|c|c|}
\hline & PRIME PLC & G3 PLC & PL360 \\
\hline $\begin{array}{l}\text { Rango de velocidad } \\
\text { (Kbps) }\end{array}$ & 130 & $5.6-46$ & Hasta 130 \\
\hline $\begin{array}{l}\text { Máxima tasa de datos } \\
\text { (Kbps) }\end{array}$ & 128.6 & 33.4 & Hasta 128.6 \\
\hline $\begin{array}{l}\text { Rango de frecuencia } \\
\text { (KHz) }\end{array}$ & $3-95$ & $3-490$ & Hasta 500 \\
\hline Solidez de ruido & Medio & Alto & Alto \\
\hline Confiablilidad & Media & Alta & Alta \\
\hline Corrección de error & Alto & Alto & Alto \\
\hline Adaptabilidad & Alto & Alto & Alto \\
\hline Rendimiento & $\begin{array}{c}\text { Dita } \\
\text { en frecuencia }\end{array}$ & $\begin{array}{c}\text { DBPSK. DQPSK } \\
\text { en tiempo }\end{array}$ & $\begin{array}{r}\text { DBPSK. DQPSK. D8PSK } \\
\text { en frecuencia } \\
\text { DBPSK. DQPSK } \\
\text { en tiempo }\end{array}$ \\
\hline $\begin{array}{l}\text { Modulación de } \\
\text { codificación }\end{array}$ & 11.52 & 16.79 & 13.32 \\
\hline Costo (USD) & & & \\
\hline
\end{tabular}

Rev. Ingeniería, Matemáticas y Ciencias de la Información Vol. 6 / Núm. 11 / enero - junio de 2019; pág. 35-44 
El modulo G3 PLC es el modulo que nos permite tener unos altos valores para ser elegido para las pruebas a realizar ya que permite una solidez del ruido alta, así como un dispositivo con una confiabilidad y una corrección de error muy alta, sería la elección más óptima para la comprobación de un sistema Smart Grid.

La implementación de la tecnología PLC en la red eléctrica permite obtener un monitoreo constante de la condición de cada uno de los usuarios en tiempo real y bastante preciso, lo que conlleva a tener una gran facilidad en la toma de decisiones y control oportuno de actividades.

El modulo PL360 es una tecnología muy reciente, el cual combina las características de las tecnologías PRIME y G3 en una sola por lo que de resultado se obtiene un modulo mucho mas eficiente y con muchas mas aplicaciones, aunque aun no tenga ninguna concreta.

\section{REFERENCIAS}

[1] J. Misurec, P. Mlynek, and S. Bezzateev, "The modeling of power line for plc in smart grids," in Progress in Electromagnetics Research SymposiumSpring (PIERS), 2017. IEEE, 2017, pp. 780-786.

[2] S. Galli, A. Scaglione, and Z. Wang, "For the grid and through the grid: The role of power line communications in the smart grid," Proceedings of the IEEE, vol. 99, no. 6, pp. 998-1027, 2011.

[3] M.Schwartz, "Carrier-wave telephony over power lines-early history," in Electric Power, 2007 IEEE Conference on the History of. IEEE, 2007, pp. 244254.

[4] M. Gotz, M. Rapp, and K. Dostert, "Power line channel characteristics and their effect on communication system design," IEEE Communications Magazine, vol. 42, no. 4, pp. 78-86, 2004.

[5] M. Tlich, A. Zeddam, F. Moulin, and F. Gauthier, "Indoor power-line communications channel characterization up to $100 \mathrm{mhz}$ - part i: oneparameter deterministic model," IEEE Transactions on Power delivery, vol. 23, no. 3, pp. 1392-1401, 2008.

[6] F. Z. Peng, J. W. McKeever and D. J. Adams, "A power line conditioner using cascade multilevel inverters for distribution systems," in IAS'97. Conference Record of the 1997 IEEE Industry Applications Conference Thirty-Second IAS Annual Meeting, vol. 2. IEEE,1997,pp.1316-1321.
[7] S. G. C. V. 2030, Antecedentes y Marco del Análisis, Evaluación y Recomendaciones para la Implementación de Redes Inteligentes en Colombia, Parte I. UPME, 2016.

[8] A. Majumder et al., "Power line communications," IEEE potentials, vol. 23, no. 4, pp. 4-8, 2004.

[9] V. H. Serna, "Comunicaciones a través de la red eléctrica-plc," Comunicaciones: Maxim FRANCE Smart Grid, 2011.

[10] P. Huang, J. Kalagnanam, R. Natarajan, D. Hammerstrom, R. Melton, M. Sharma, and R. Ambrosio, "Analytics and transactive control design for the pacific northwest smart grid demonstration project," in Smart Grid Communications (SmartGridComm), 2010 First IEEE International Conference on. IEEE, 2010, pp. 449454.

[11] W. Bartel, “Houston's smart grid: Transforming the future of electric distribution \& energy consumption," 2012.

[12] I. Global Industry Analysts, Power Line Communication (PLC) Systems - Global Strategic Business Report. Global, 2018.

[13] V. Giordano, F. Gangale, G. Fulli, M. S. Jiménez, I. Onyeji, A. Colta, I. Papaioannou, A. Mengolini, C. Alecu, T. Ojala et al., "Smart grid projects in europe," JRC Ref Rep Sy, vol. 8, 2011.

[14] C. de Antonio, Y. Bladymir et al., "Estudio de la factibilidad técnica de la implementación de plc (power line communication) en la red de distribución eléctrica de bogotá," Ph.D. dissertation, Universidad Nacional de Colombia-Sede Bogotá.

[15] S. G. C. V. 2030, Antecedentes y Marco del Análisis, Evaluación y Recomendaciones para la Implementación de Redes Inteligentes en Colombia, Parte II. UPME, 2016.

[16] J.-J. Lee, S.-J. Choi, H.-M. Oh, W.-T. Lee, K.-H. Kim, and D.-Y. Lee, "Measurements of the communications environment in medium voltage power distribution lines for wide-band power line communications," in Proc. of, 2004, pp. 69-74.

[17] D. E. Nordell, "Communication systems for distribution automation," in Transmission and Distribution Conference and Exposition, 2008. T\&D. IEEE/PES. IEEE, 2008, pp. 1-14.

[18] S. T. Mak and D. L. Reed, "Twacs, a new viable two-way automatic communication system for distribution networks. part i: Outbound communication," IEEE Transactions on Power Apparatus and systems, no. 8, pp. 2941-2949, 1982.

[19] S. T. Mak and T. G. Moore, "TwacsTM, a new viable two-way automatic communication system for 
distribution networks. part ii: Inbound communication," IEEE transactions on power apparatus and systems, no. 8, 1984.

[20] R. Perlman, C. Kaufman, and M. Speciner, Network security: private communication in a public world. Pearson Education India, 2016.

[21] B. Fung, A. Khitrin, and K. Ermolaev, “An improved broadband decoupling sequence for liquid crystals and solids," Journal of Magnetic Resonance, vol. 142, no. 1, pp. 97-101, 2000.

[22] K. Sharma and L. M. Saini, "Power-line communications for smart grid: Progress, challenges, opportunities and status," Renewable and Sustainable Energy Reviews, vol. 67, pp. 704-751, 2017.

[23] S. A. Boyer, SCADA: supervisory control and data acquisition. International Society of Automation, 2009.

[24] J. C. Cuevas, J. Martínez, and P. Merino, “El protocolo x10: una solución antigua a problemas actuales," in Simposio de informática y telecomunicaciones SIT, 2002.

[25] G. W. Pfleging, R. M. Pfleging, and G. P. Wilkin, "X10 communication of one or more messages between one or more mobile communication devices and one or more module components," Aug. 15 2006, uS Patent 7,091,853.

[26] L. T. Berger, A. Schwager, and J. J. Escudero-Garzás, "Power line communications for smart grid applications," Journal of Electrical and Computer Engineering, vol. 2013, p. 3, 2013.

[27] S. Moaveninejad, A. Kumar, D. Scazzoli, A. Piti, M. Magarini, S. Bregni, and G. Verticale, "Ber evaluation of post-meter plc services in cenelec-c band," in Communications (LATINCOM), 2017 IEEE 9th Latin-American Conference on. IEEE, 2017, pp. 1-6.

[28] H. P. Alliance, "Homeplug av white paper," http:/ /www. homeplug. org/products/whitepapers/, 2007.

[29] S. Galliand T. Lys, "Next generation narrow band (under 500khz) power line communications (plc) standards," China Communications, vol. 12, no. 3, pp. 1-8, 2015.

[30] H. C. Ferreira, L. Lampe, J. Newbury, and T. G. Swart, Power line communications: theory and applications for narrowband and broad- band communications over power lines. John Wiley \& Sons, 2011.
[31] Z. Fan, P. Kulkarni, S. Gormus, C. Efthymiou, G. Kalogridis, M. Sooriyabandara, Z. Zhu, S. Lambotharan, and W. H. Chin, "Smart grid communications: Overview of research challenges, solutions, and standardization activities," IEEE Communications Surveys \& Tutorials, vol. 15, no. 1, pp. 21-38, 2013.

[32] V. C. Gungor, D. Sahin, T. Kocak, S. Ergut, C. Buccella, C. Cecati, and G. P. Hancke, "Smart grid technologies: Communication technologies and standards," IEEE transactions on Industrial informatics, vol. 7, no. 4, pp. 529-539, 2011.

[33] G. López, J. Moreno, H. Amarís, and F. Salazar, "Paving the road toward smart grids through large-scale advanced metering infrastructures," Electric Power Systems Research, vol. 120, pp. 194205, 2015.

[34] V. Oksman and S. Galli, “G. hn: The new itu-t home networking standard," IEEE Communications Magazine, vol. 47, no. 10, 2009.

[35] J. LeClare, A. Niktash, and V. Levi, "An overview, history, and formation of ieee p1901. 2 for narrowband ofdm plc," Maxim Integrated, Application Note, vol. 5676, pp. 1-7.

[36] L. Yonge, J. Abad, K. Afkhamie, L. Guerrieri, S. Katar, H. Lioe, P. Pagani, R. Riva, D. M. Schneider, and A. Schwager, "An overview of the homeplug av2 technology," Journal of Electrical and Computer Engineering, vol. 2013, 2013.

[37] M. M. Rahman, C. S. Hong, S. Lee, J. Lee, M. A. Razzaque, and J. H. Kim, "Medium access control for power line communications: an overview of the ieee 1901 and itu-t g. hn standards," IEEE Communications Magazine, vol. 49, no. 6, 2011.

[38] Z. Sadowski, "Comparison of plc-prime and plcg3 protocols," in Nonsinusoidal Currents and Compensation (ISNCC), 2015 International School on. IEEE, 2015, pp. 1-6.

[39] M. Hoch, "Comparison of plc g3 and prime," in Power Line Communications and Its Applications (ISPLC), 2011 IEEE International Symposium on. IEEE, 2011, pp. 165-169.

[40] T. Microchip, PL360 Datasheet. Microchip Technology Inc, 2018. 